\title{
LA ESCUELA AZUL DE FALANGE ESPAÑOLA DE LAS J.O.N.S. UN PROYECTO FASCISTA DESMANTELADO POR IMPLOSIÓN
}

por José Ramón López Bausela, Santander - Madrid: Editorial de la Universidad de Cantabria - Dykinson, 2017, 353 páginas. ISBN: 978-84-9148-150-8.

\section{PERO... ¿EXISTIÓ ALGUNA VEZ UN PROGRAMA EDUCATIVO FASCISTA?}

Los planteamientos educativos del falangismo han suscitado poco interés en la historiografía educativa española. Esta escasa atención resulta cuanto menos sorprendente si se tiene en cuenta que el falangismo fue uno de los pilares políticos de la dictadura franquista y el enorme poder que tuvo el partido único que controlaba. A pesar de estudios puntuales sobre algunas figuras claves del falangismo educativo ${ }^{1}$ o de mis trabajos sobre su intervención en la política educativa sobre la enseñanza media, ${ }^{2}$ se entiende que en el reparto de áreas de influencia del régimen la educación correspondió a los católicos y que, por tanto, la incidencia educativa del falangismo habría sido meramente epidérmica en el sistema formal, a través de las asignaturas de formación política y educación física, y en todo caso restringida a la educación no formal, a través de las organizaciones juveniles. De hecho, disponemos de múltiples estudios sobre la educación física y de los excelentes trabajos de José Ignacio Cruz Orozco

\footnotetext{
${ }^{1}$ Juan Mainer Baqué y Julio Mateos Montero, Saber, poder y servicio. Un pedagogo orgánico de Estado: Adolfo Maíllo (Valencia: Tirant lo Blanch, 2011). Gustavo Alares, Nacional-sindicalismo e Historia. El archivo privado de José Navarro Latorre (1916-1986) (Zaragoza, Institución Fernando el Católico, 2015).

${ }^{2}$ Antonio Fco. Canales Serrano. «Falange y Educación: El SEPEM y el debate sobre el Bachillerato en los años cuarenta», Educación XXI, 15 (1), (2012): 219-239, y «Falangistas contra la hegemonía educativa católica. El SEPEM y la movilización falangista contra la Ley de Bachillerato de 1938 (1942-1953)», en Ayeres en construcción. Temas Claves de Historia Contemporánea hoy, eds. Encarna Nicolás y Carmen González Marín (Murcia, Universidad de Murcia, 2008).
} 
sobre las organizaciones juveniles. ${ }^{3}$ Pero más allá de estos ámbitos, académicamente subvalorados y popularmente trivializados, se acepta que los falangistas poco dijeron, y menos hicieron, en el núcleo duro del sistema educativo, es decir, en la práctica habitual de los centros educativos.

La publicación del libro de José Ramón López Bausela habría de ser saludada con alborozo aunque sólo fuera por su contribución a la superación de este vacío. El trabajo tiene como objetivo el estudio de las posiciones educativas del falangismo y se circunscribe a un periodo muy limitado: el último año y medio republicano y los primeros meses de la guerra civil. Estos límites cronológicos vienen determinados por los dos pilares documentales sobre los que López Bausela levanta su obra: el informe del jefe de profesorado de Falange Española, Benito Sanz Marco de febrero de 1935 y el reglamento de la organización falangista de maestros (SEM) de mayo del mismo año, de un lado, y, de otro, la documentación del Consejo Nacional de Educación falangista de diciembre de 1936. Se trata en ambos casos de fuentes inéditas, de un indudable valor y de gran trascendencia. Las primeras cuestionan la idea de que el falangismo previo a la guerra no tuviera un ideario pedagógico, y además resitúan el nacimiento del SEM en Madrid, en lugar de en Zaragoza, y un año antes de lo aceptado habitualmente. La segunda fuente permite un análisis detallado de la propuesta falangista de reorganización de la totalidad del sistema educativo en un momento en que el movimiento se encontraba en la cresta de la ola en tanto que principal vehículo de la movilización popular al servicio de los sublevados. El autor salva el espacio entre ambas fuentes con un recorrido por diferentes publicaciones locales de los primeros meses de la guerra a la búsqueda de escritos falangistas que permitan avanzar en la caracterización de las ideas del movimiento sobre la educación. En un último capítulo extiende este estudio a lo largo de los primeros meses de 1937. Señala así López Bausela una de las vías de desarrollo más prometedora, pero apenas transitada, para la historiografía educativa sobre el franquismo: el estudio descentralizado de la prensa falangista. Sería deseable contar, como en otros ámbitos de la historiografía sobre el régimen, con estudios parciales y limitados sobre lo que a

\footnotetext{
${ }^{3}$ José I. Cruz Orozco, El yunque azul. Frente de Juventudes y sistema educativo: razones de un fracaso (Madrid: Alianza editorial, 2001) y Prietas las filas. Las falanges Juveniles de Franco (Valencia, Universitat de València, 2012).
} 
lo largo y ancho del territorio rebelde los falangistas entendían en vivo y en directo que había de constituir la nueva educación.

El armazón estructural que sostiene la obra de López Bausela queda constituido, pues, por el análisis detallado de la documentación inédita de dos periodos concretos conectados por una aproximación necesariamente asistemática a la prensa falangista de los primeros meses de la guerra. Nada que objetar a esta estructura que bastaría por sí misma para justificar la oportunidad e idoneidad de una obra que, como se indicó con anterioridad, contribuye a llenar un vacío absoluto a través de una valiosa documentación inédita. Hasta este punto, el libro no puede merecer más que unánimes elogios. Los problemas comienzan cuando el autor pretende ir más allá del estudio de esta documentación concreta completando su contribución empírica con una tesis teórica que defiende la existencia de un proyecto de escuela propiamente falangista contrapuesto de manera significativa al proyecto nacional-católico que acabó imponiéndose.

Este salto teórico supone, sin lugar a dudas, un planteamiento más ambicioso, y lucido, que el mero análisis empírico, pero también mucho más arriesgado, pues son múltiples las objeciones que cabe oponerle. La primera cuestionaría si la limitada base documental trabajada, por muy importante que sea, permite realmente fundamentar una tesis de ese alcance. En realidad, todo se cimenta sobre las conclusiones de un Consejo y una aproximación general y asistemática a la prensa, nada más. La segunda objeción se derivaría de una cierta inconsistencia entre la cronología del proceso y la tesis defendida. El autor no concluye su estudio con la documentación del Consejo Nacional de Educación de 1936, como cabría esperar desde un planteamiento ajustado a la fuentes disponibles, sino que lo alarga con un nuevo repaso asistemático a la prensa para llegar hasta abril 1937. Este alargamiento, que fuerza la simetría estructural de la obra y le resta elegancia, le permite identificar su trabajo con el estudio de la Falange auténtica previa a su domesticación por Franco a través de la unificación. La razón de que las conclusiones del Consejo Nacional de Educación falangista nunca se publicaran estribaría entonces en la voluntad del régimen de diluir y desvirtuar el proyecto que contenían, tarea de la que se haría cargo Pedro Sainz Rodríguez, primero como delegado nacional de educación del partido único y más tarde 
como ministro de educación. Ahora bien, existe en este planteamiento una incongruencia cronológica que el capítulo final añadido ad hoc no consigue ocultar: el Consejo de celebró en diciembre de 1936 y la Unificación no se produjo hasta mediados de abril de 1937. Los falangistas dispusieron, pues, de nada menos que de cuatro preciosos meses en el trepidante tempo bélico para hacer públicos sus planteamientos antes de su domesticación por el régimen. La atribución unívoca de esta omisión a una intervención externa posterior no parece especialmente convincente; ¿por qué no a las vacilaciones y reticencias dentro del propio partido? La recepción de la propuesta en el resto del partido es un tema ignorado por el autor, a pesar de que la trascendencia de la cuestión y su carácter potencialmente conflictivo con los católicos no hace plausible que la cúpula dirigente se desentendiera de ella.

Más allá de estas cuestiones inherentes al estudio de López Bausela, existen otras dificultades de ámbito general derivadas del marco teórico en que el autor inserta su planteamiento. La obra se sitúa en la tesis de los dos componentes fundamentales del franquismo: el nacional-catolicismo, tradicionalista en sus planteamientos esenciales, y el falangismo, auténticamente fascista y moderno, que constituirían los dos polos de tensión en torno a los que se habría construido y desarrollado el régimen. Se trata de un modelo que intenta responder al desafío planteado por la pluralidad de fuentes ideológicas, y para algunos también de proyectos políticos, que convergieron en el franquismo; una heterogeneidad que dio lugar a la polémica tesis del pluralismo limitado de Juan Linz en los años sesenta, y que ha sido reformulada magistralmente por Ismael Saz para los años iniciales del régimen en las últimas dos décadas. ${ }^{4}$ Paradójicamente, en este planteamiento actual encaja bastante bien el principal marco interpretativo de la educación bajo el franquismo que sigue siendo el excelente libro de Gregorio Cámara Villar publicado nada menos que en $1984 .{ }^{5}$ Como se señaló más arriba, parece comúnmente aceptado que los católicos se hicieron con el control de la educación y que la Falange quedó al margen. De esta manera, la historiografía educativa vendría a converger con la historiografía más actual sobre el franquismo... ¡isin

\footnotetext{
${ }^{4}$ Ismael Saz Campos, España contra España. Los nacionalismos franquistas (Madrid: Marcial-Pons, 2003).

${ }^{5}$ Gregorio Cámara Villar, Nacional-catolicismo y escuela: la socialización política del franquismo, 1936-1951 (Jaén, Hesperia, 1984).
} 
apenas haberse movido en tres décadas!. Y López Bausela abona esta tesis estudiando el periodo anterior a la domesticación de Falange, para establecer la existencia en términos fuertes de un proyecto falangista diferenciado que posteriormente sería desvirtuado en favor de los católicos.

La tesis historiográfica de partida, sin embargo, está lejos de constituir la panacea para la resolución de los problemas interpretativos del franquismo. De un lado, se trata de una contraposición de tipos ideales, que sin duda tiene una innegable potencialidad heurística a la hora de abordar la heterogeneidad de posiciones ideológicas y políticas que integraron el franquismo, pero que no por ello deja de suponer una idealización simplificada de la realidad. Sobre el terreno, las cosas eran mucho más complejas. La dificultad para la aplicación práctica del modelo no proviene únicamente de la ardua tarea de distinguir entre un católico fascistizado y un fascista católico, sino de la complicación casi insalvable que añade la existencia de nacional-católicos disfrazados instrumentalmente de fascistas y de falangistas que, a pesar de su tibieza religiosa, comulgaban entusiásticamente con el relato imperial nacional-católico. De hecho, el ámbito de la educación supone un espacio privilegiado para constatar esta complejidad plagada de ambigüedades, puesto que algunas de las figuras más relevantes del nacional-catolicismo fascistoide o fascistizado se movieron precisamente en el Ministerio de Educación Nacional, como fue el caso de José Pemartín o el del propio Sainz Rodríguez. En otros lugares he planteado la dificultad de reducir a estos hombres simplemente a católicos que instrumentalizaron el fascismo; ${ }^{6}$ pero más allá de esta cúpula dirigente ¿cómo calificar a hombres clave, aunque poco conocidos, como los inspectores que se erigieron en un primer momento en portavoces del pensamiento pedagógico franquista? Podríamos aceptar que Antonio J. Onieva era un fascista vitalista y que Agustín Serrano de Haro un prototípico católico fascistizado, pero ¿qué hacer con Alfonso Iniesta? Tras su loa de las organizaciones juveniles en El orden nuevo en la educación de juventudes de 1941, ¿podemos aceptar en serio que su asunción del fascismo era meramente instrumental?

Esta dificultad para definir proyectos diferenciados planea continuamente sobre el análisis que López Bausela realiza de los discursos educa-

\footnotetext{
${ }^{6}$ Antonio Fco. Canales Serrano, «Pemartín y la frustrada fascistización de la enseñanza media de posguerra», Historia Social, 74 (2012): 65-84.
} 
tivos falangistas. En contra de la interpretación del autor, el propio José Antonio parecía comulgar más con los planteamientos básicos del nacional-catolicismo educativo que esbozar algo alternativo: catolicismo, rechazo a Rousseau, «hombre nefasto» (p. 57), nostalgia del Imperio e interpretación nacional-católica de la historia de España (p. 58) y preocupación por «el sentido cristiano y nacional» de las escuelas (p. 63). ¿Proyecto propio o más bien combinación particular de acentos?

Tampoco el análisis de los documentos de 1935 y de los artículos de prensa de los primeros meses de la guerra parece apuntar mucho más allá de una particular combinación de acentos. Clamaban los falangistas en la prensa contra los pedagogías extranjerizantes, reclamaban el retorno a una pedagogía verdaderamente española de raíz cristiana y abominaban de «la nunca bastante maldita» Institución Libre de Enseñanza (p. 240). Afirmaciones todas ellas que suscribirían sin reticencia alguna tradicionalistas de rancia raigambre como Romualdo de Toledo, máxime cuando la alternativa propuesta a Claparède, Decroly, Dewey o Ferrière eran el Padre Manjón o San José de Calasanz (p. 253). Ciertamente, ya en los documentos de 1935 los falangistas introducían elementos nuevos como la extrema jerarquización y el encuadramiento de profesorado y alumnado. No obstante, esas novedades no se derivaban tanto de un posicionamiento pedagógico diferenciado como de su condición de fascistas, de la misma manera que la aspiración a «reconstruir, empezando por los cimientos, todo el cochambroso edificio pedagógico, educativo» (p. 129) no es más que una muestra de la característica palingenesia fascista. Si no hablaran de jerarquía, encuadramiento y palingenesia los falangistas simplemente no serían fascistas, y no dudamos de que lo fueran. La cuestión fundamental es qué había de específico en su programa educativo más allá de estos elementos constitutivos del fascismo. Y me atrevería a responder que poco.

Un repaso a los principios que inspiraban el proyecto educativo falangista de diciembre de 1936 parece avalar esta impresión. Elementos como la centralidad del catolicismo o el rechazo a la coeducación subrayan la identidad de fondo con el proyecto católico más tradicional, al igual que la aproximación a la educación femenina, en contra de lo que defiende el autor. Ciertamente, el fascismo defendía el retorno de la mujer a sus funciones tradicionales y la introducción de un currícu- 
lo diferenciado congruente con ellas como la economía doméstica, pero esta concepción no era privativa del fascismo, sino un préstamo de las concepciones católicas y conservadoras tradicionales. En contraposición a estas posiciones tradicionales, su condición de fascistas llevaba a los falangistas a introducir algunas novedades como la extrema jerarquización de la inspección educativa, la educación física entendida en términos de formación premilitar (aunque luego se olvidasen de ella en su plan de estudios), o las declaraciones sociales de no perder ningún talento por razones económicas y abrir la educación a todos los sectores sociales. En este sentido, la propuesta de escuela postelemental ilustra a la perfección la provisionalidad e indefinición en la aplicación de estos principios. Los falangistas proponían una ampliación de la escolarización obligatoria hasta los 14 años y la gratuidad hasta los 16 a través de escuela primaria superior y una escuela técnica, vagamente definidas, que el autor conecta con el fascismo italiano, aunque debe reconocer que en realidad parecen más bien de inspiración republicana francesa, modelo que los españoles, falangistas o no, conocían mucho mejor que el caso italiano... y no digamos el alemán.

A pesar de su vaguedad, estas propuestas ligadas a la organización estructural de una enseñanza ampliada constituyen los elementos más originales del falangismo frente al tradicional programa educativo católico. Así, resulta muy interesante su apuesta por la escuela graduada, estandarte y talismán de la renovación pedagógica española. Pero seguramente lo más original y novedoso del programa falangista parece haber sido su apuesta radical por un cuerpo del magisterio autosuficiente y jerarquizado que cubriera desde la escuela rural hasta la inspección, pasando por la dirección de las escuelas graduadas y la enseñanza en las escuelas normales. Con esta propuesta, el falangismo se opondría drásticamente al proceso de separación entre científicos universitarios (pedagogos) llamados a actuar como directivos y los trabajadores de a pie (maestros) que se había iniciado con la creación de una Sección de Pedagogía universitaria bajo la República y que se consolidaría durante el franquismo, un proceso que describe magistralmente Juan Mainer Baqué. ${ }^{7}$ Igualmente interesante resulta la propuesta de plan de estudios

\footnotetext{
${ }^{7}$ Juan Mainer Baqué, La forja de un campo profesional. Pedagogía y didáctica de las ciencias sociales en España (1900-1970) (Madrid: CSIC, 2009).
} 
para la escuela primaria que recoge en gran medida los planteamientos escolanovistas de Onieva sobre el niño como punto de partida del currículo. No cabe duda de que las páginas dedicadas al estudio de las propuestas de la asamblea falangista de diciembre de 1936 son la parte más interesante del libro y justifican por sí mismas su publicación. Otra cosa es que fundamenten de manera convincente la tesis de la existencia de un proyecto alternativo en un sentido fuerte.

Un último escollo al que se enfrenta la argumentación del autor en favor de esta tesis es el recurso como referencia a los casos de Alemania e Italia. Los problemas derivados de la complejidad, transversalidad y heterogeneidad no se limitaban al caso español, sino que eran inherentes a los fascismos prototípicos. Los fascistas no surgieron de la nada por generación espontánea, sino de un complejo proceso de evolución y mutación de tradiciones existentes, y desde luego no eran monolíticos en sus planteamientos, y mucho menos en el ámbito educativo (algo pensarían sobre educación antes de hacerse fascistas y en algún lugar se habrían formado). Por ello el recurso del autor a los casos italiano y alemán no hace más que subrayar la idealización que subyace a todo el planteamiento. López Bausela se ampara en dos ocasiones en la misma cita de un artículo del reconocido investigador Francisco Morente que afirma que los fascismos contaban «con una base teórica en materia de educación más que sólida» ${ }^{8}$ (pp. 67 y 181). No obstante, no sea esta quizás la cita más afortunada para captar el planteamiento del excelente artículo de Morente, quien dedica las páginas siguientes a ilustrar lo contrario, es decir, los préstamos y alianzas del fascismo en materia educativa. Para el caso italiano, Morente defiende en varios lugares que el fascismo no tenía un programa educativo, y que se alió inicialmente con el actualismo, una corriente pedagógica sólidamente desarrollada y liderada por el filósofo idealista Gentile, quien sería el primer ministro de educación de Mussolini y el responsable de la gran reforma educativa de $1923 .{ }^{9}$ Pero fue una alianza inicial, pues tanto el actualismo como la reforma que inspiró fueron duramente criticados y cuestionados por los fascistas en los años siguientes. En todo caso, el actualismo pactó con el fascismo, pero

\footnotetext{
${ }^{8}$ Francisco Morente Valero, «Los fascismos europeos y la política educativa del franquismo», Historia de la Educación, 24, (2005): 182.

${ }^{9}$ Véase también Francisco Morente Valero, «Libro e Moschetto». Política educativa y política de juventud en la Italia fascista (Barcelona: PPU, 2001).
} 
no era un proyecto educativo fascista, ni mucho menos el proyecto educativo fascista. De la misma manera, Lombardo Radice, director general de enseñanza primaria con Gentile, al que el autor toma como referencia en varias ocasiones, no era un fascista, sino un pedagogo renovador de inspiración socialista que se distanció rápidamente del fascismo. Y algo similar señala Morente sobre la Alemania nazi, pues nunca habla en el artículo citado de la existencia de una pedagogía nazi, sino de su «indigencia en materia pedagógica» y del acomodo al nazismo de diferentes tradiciones pedagógicas, ${ }^{10}$ que es cosa muy diferente. La referencia a los casos prototípicos, por tanto, no ayuda en absoluto a definir lo que era un proyecto educativo fascista, sino más bien lo contrario. Y es que la pregunta fundamental, tanto en España como en el resto del mundo, es si existió alguna vez un programa educativo fascista más allá de la extrema ideologización, la jerarquización y el encuadramiento de alumnado y profesorado.

Desde mi punto de vista no estamos en condiciones de dar una respuesta a esta pregunta. La mejor manera de avanzar hacia una respuesta no es desde luego perseverar en la idealización, sino, por el contrario, zambullirnos de lleno en la investigación documental para avanzar de manera significativa en nuestro conocimiento positivo de las posiciones educativas de los fascistas. Este programa no supone un abandono al craso positivismo, sino una apuesta por una investigación empírica colectiva de largo alcance que requiere en primer lugar de la realización de un mapa del debate educativo que identifique las principales cuestiones que articulaban la discusión para más tarde caracterizar y evaluar el posicionamiento fascista en cada uno de los temas. Si es posible identificar una combinación de respuestas educativas comunes a los fascistas, estaremos sin duda ante la anhelada caracterización del Proyecto Educativo Fascista con mayúsculas. Si no es así, no nos quedará otra que conformarnos con programas específicos para cada país, en el caso de que la combinación de respuestas sea realmente original, o simplemente con una modulación al hilo de los principios fascistas de discursos compartidos, como defiendo para el caso español.

En todo caso, esta propuesta resulta una vía mucho más operativa para el estudio del falangismo educativo que la contraposición idealista.

\footnotetext{
${ }^{10}$ Morente Valero, «Los fascismos europeos», 185.
} 
Resulta necesario reconstruir el debate educativo español del primer tercio del siglo xx por encima del hegemónico relato idealista que acertadamente critica Juan Mainer Baqué ${ }^{11}$ para constatar qué puntos se estaban debatiendo en realidad y cuál era la postura de cada cual. No cabe duda de que las ventajas derivadas de este ejercicio no se reducirían únicamente a los falangistas, sino que ayudarían a perfilar en gran medida las posiciones del resto de agentes participantes, como los propios católicos de los que no sabemos tanto como creemos.

Es precisamente desde esta perspectiva que apuesta por la investigación empírica que quedan patentes tanto la importancia y el extraordinario interés de la obra de López Bausela como la necesidad de que otros trabajos completen sus hallazgos. En última instancia, la tesis del autor de la existencia de un programa educativo falangista en un sentido fuerte que he venido discutiendo en los párrafos anteriores no es en absoluto una tesis errónea, sino simplemente una tesis prematura. Por ello, no cabe más que felicitar a López Bausela por este libro y esperar que los/as jóvenes investigadores/as perciban el atractivo del camino que abre.

Antonio Fco. Canales Serrano

Universidad de La Laguna acanales@ull.edu.es

${ }^{11}$ Juan Mainer Baqué, «De largas noches y rupturas lampedusianas. Franquismo y relato canónico de la educación española». Con-Ciencia Social, 20 (2016): 143-150. 